\title{
Real-World Experience with Insertable Cardiac Monitors to Find Atrial Fibrillation in Cryptogenic Stroke
}

\author{
Paul D. Ziegler $^{\mathrm{a}}$ John D. Rogers ${ }^{\mathrm{b}}$ Scott W. Ferreira $^{c}$ Allan J. Nichols ${ }^{\mathrm{d}}$ \\ Shantanu Sarkar $^{\mathrm{a}}$ Jodi L. Koehler ${ }^{\mathrm{a}}$ Eduardo N. Warman ${ }^{\mathrm{a}}$ Mark Richards ${ }^{\mathrm{e}}$ \\ a Medtronic Cardiac Rhythm Heart Failure, Mounds View, Minn., b Scripps Clinic, La Jolla, Calif., cSt. Louis University, \\ St. Louis, Mo., dOhio Health Heart and Vascular Physicians, Columbus, Ohio, eProMedica Physicians Cardiology, \\ Toledo, Ohio, USA
}

\section{Key Words}

Atrial fibrillation · Cryptogenic stroke · Insertable cardiac monitor $\cdot$ Stroke assessment $\cdot$ Stroke (ischemic)

\begin{abstract}
Background: The characteristics of atrial fibrillation (AF) episodes in cryptogenic stroke patients have recently been explored in carefully selected patient populations. However, the incidence of AF among a large, real-world population of patients with an insertable cardiac monitor (ICM) placed for the detection of AF following a cryptogenic stroke has not been investigated. Methods: Patients in the de-identified Medtronic DiscoveryLink ${ }^{\mathrm{TM}}$ database who received an ICM (Reveal LINQ ${ }^{\mathrm{TM}}$ ) for the purpose of AF detection following a cryptogenic stroke were included. AF detection rates (episodes $\geq 2 \mathrm{~min}$ ) were quantified using Kaplan-Meier survival estimates at 1 and 6 months and compared to the CRYSTAL AF study at 6 months. The time to AF detection and maximum duration of AF episodes were also analyzed. Results: A total of 1,247 patients (age $65.3 \pm 13.0$ years) were followed for 182 (IQR 182-182) days. A total of 1,521 AF episodes were detected in 147 patients, resulting in AF detection rates of 4.6 and $12.2 \%$ at 30 and 182 days, respectively, and representing a $37 \%$ relative increase over that reported in the CRYSTAL AF trial at 6 months. The median time to AF detec-
\end{abstract}

tion was 58 (IQR 11-101) days and the median duration of the longest detected AF episode was 3.4 (IQR 0.4-11.8) h. Conclusions: The real-world incidence of AF among patients being monitored with an ICM after a cryptogenic stroke validates the findings of the CRYSTAL AF trial and suggests that continuous cardiac rhythm monitoring for periods longer than the current guideline recommendation of 30 days may be warranted in the evaluation of patients with cryptogenic stroke.

(C) 2015 The Author(s)

Published by S. Karger AG, Basel

\section{Introduction}

Each year in the United States, an estimated 675,750 patients experience an ischemic stroke at some stage of their lives [1]. However, the causes of these strokes remain undetermined in up to $30 \%$ of cases and subsequently these events are designated as cryptogenic [2]. Since atrial fibrillation (AF) can frequently be intermittent and asymptomatic [3-5], it is often missed by sporadic or brief periods of arrhythmia monitoring $[6,7]$. Consequently, the opportunity to treat AF with oral anticoagulation to reduce the risk of recurrent stroke may also be missed [8-11]. 
Current stroke guidelines now recommend up to 30 days of cardiac rhythm monitoring for patients who have experienced an acute ischemic stroke or TIA with no other apparent cause [12]. However, numerous small, singlecenter studies have demonstrated that significant proportions of patients with strokes initially believed to be cryptogenic actually have episodes of occult AF detected beyond 30 days of monitoring via even more extensive rhythm surveillance with insertable cardiac monitors (ICMs) [13-17].

The incidence and duration of AF episodes detected by ICMs in cryptogenic stroke patients have recently been explored in a multicenter clinical trial of carefully selected patients [18]. Our goal was to investigate the incidence and duration of AF episodes among a much larger, realworld population of unselected patients with ICMs placed for the detection of AF following a cryptogenic stroke in clinical practice.

\section{Methods}

Patients in the de-identified Medtronic Discovery ${ }^{\mathrm{TM}}$ Link database who received an ICM (Reveal LINQ ${ }^{\mathrm{TM}}$, Medtronic, Minn., USA) for the purpose of AF detection following a cryptogenic stroke were included in this study. The implanting physician indicated that cryptogenic stroke was the reason for monitoring at the time of initial device programming. Patients were monitored for up to 182 days following the date of device insertion. All patients provided consent to use their device data for research purposes.

\section{Device Insertion and Capabilities}

The Reveal LINQ ${ }^{\mathrm{TM}}$ ICM system consists of an insertion kit, the Reveal LINQ ${ }^{\mathrm{TM}}$ device, and the MyCareLink ${ }^{\mathrm{TM}}$ patient monitor to permit remote transmission of device data. Insertion of the device is facilitated by dedicated incision and insertion tools. The incision tool safely creates a small opening in the skin, which is sized precisely for the dimensions of the ICM. The insertion tool assists in the creation of the pocket and placement of the device in the subcutaneous tissue. Devices were inserted subcutaneously with a recommended position of 45 degrees relative to the sternum over the 4 th intercostal space (V2-V3 electrode orientation). The superior end of the device is positioned approximately $2 \mathrm{~cm}( \pm 1 \mathrm{~cm})$ left lateral from the sternal border. Alternatively, the device may be positioned over the 4 th intercostal space approximately $2 \mathrm{~cm}$ $( \pm 1 \mathrm{~cm})$ parallel to the sternal border.

The Reveal LINQTM ICM measures $44.8 \mathrm{~mm}$ long by $7.2 \mathrm{~mm}$ wide by $4.0 \mathrm{~mm}$ thick, has a volume of $1.2 \mathrm{~cm}^{3}$, and weighs $2.5 \mathrm{~g}$. Electrodes on the ends of the device record ECG signals and an embedded accelerometer measures patient activity. The device has a dedicated AF detection algorithm (described below) from which the incidence and duration of AF episodes can be determined. The device can store up to $14 \mathrm{AF}$ episodes with electrocardiogram (ECG) data after which the earliest episode gets overwritten by newer episodes. Each day the ICM also tabulates the cumulative time spent in $\mathrm{AF}$ (AF burden) as well as ventricular rate during AF, average day and night heart rate, heart rate variability, and patient activity.

\section{Data Transmission}

Data from the ICMs were obtained through remote telemetry from the patient's residence, permitting daily transfer of the device diagnostic data to the Medtronic CareLink ${ }^{\circledR}$ data server. When an AF episode is detected, the first 2 min of ECG from that episode is stored in the device. The longest detected AF episode $\geq 10 \mathrm{~min}$ in duration is always preserved in memory until a full manual transmission is performed. The patient can manually transmit full information for all AF episodes stored in memory at any given time. Additionally, every night the device automatically attempts to wirelessly transmit the last $10 \mathrm{~s}$ of the initial 2-minute ECG segment for the longest AF episode during the previous day. The de-identified DiscoveryLink ${ }^{\mathrm{TM}}$ database was created from the stored data of devices implanted in the United States. Parameters included in the analysis were age, gender, and individual AF episode characteristics (date of onset, episode duration, and ECG waveforms).

\section{AF Detection}

Details of the AF detection algorithm have been previously described [19]. In brief, the algorithm in the Reveal LINQ ICM evaluates the irregularity and incoherence of ventricular conduction patterns over a 2-minute analysis window by comparing changes in R-R intervals between successive beats. The AF detection algorithm makes rhythm decisions every 2 min and consequently, a minimum episode duration of 2 min was utilized in this analysis.

This algorithm has been previously validated against simultaneous Holter monitor data in patients with known AF as part of the XPECT study and was shown to have a sensitivity of $96.1 \%$ for identifying patients with AF and a negative predictive value of $97.4 \%$ for correctly excluding the absence of AF [20]. Subsequent enhancements to the AF detection algorithm were included in the Reveal LINQ device to reduce the duration and number of false positive detections by 55 and $46 \%$, respectively, without affecting algorithm sensitivity [21].

All episodes collected by the device during the first 6 months after implant that were stored with either a 10 s or 2 min ECG segment were reviewed by a single scientific reviewer (S.S.) who was blinded to all patient characteristics. All episodes that were annotated to be true and an equal number of randomly selected episodes annotated to be false from the initial review were reviewed a second time to validate the episode annotation process by ensuring at least 99\% agreement. Episodes were considered to be true if there was at least a $10 \mathrm{~s}$ ECG segment showing an atrial arrhythmia and were considered to be either true or false for their entire duration based on the $10 \mathrm{~s}$ or $2 \mathrm{~min}$ ECG segment stored at the onset of the episode.

\section{Statistical Analysis}

AF detection rates were quantified at 1 month (to allow comparison to the current guideline recommendations for monitoring) and 6 months (to allow comparison to the primary endpoint of CRYSTAL AF) using Kaplan-Meier survival estimates for minimum episode duration thresholds of 2, 6, 30, and $60 \mathrm{~min}$. We also analyzed the median time to detection of the first adjudicated AF episode and the median duration of the longest AF episode for patients with AF detected. The age of patients with vs. without AF detected was compared with the t test and age was also compared between patients with varying maximum AF episode durations $(<1,1-4,4-12$, and $>12 \mathrm{~h})$ using an ANOVA model. The AF detection rate for patients $\leq 60$ years of age was compared to patients $>60$ years of age using the log-rank test. 
Fig. 1. Time to detection of AF using episode duration thresholds of 2, 6, 30, and 60 $\min$.
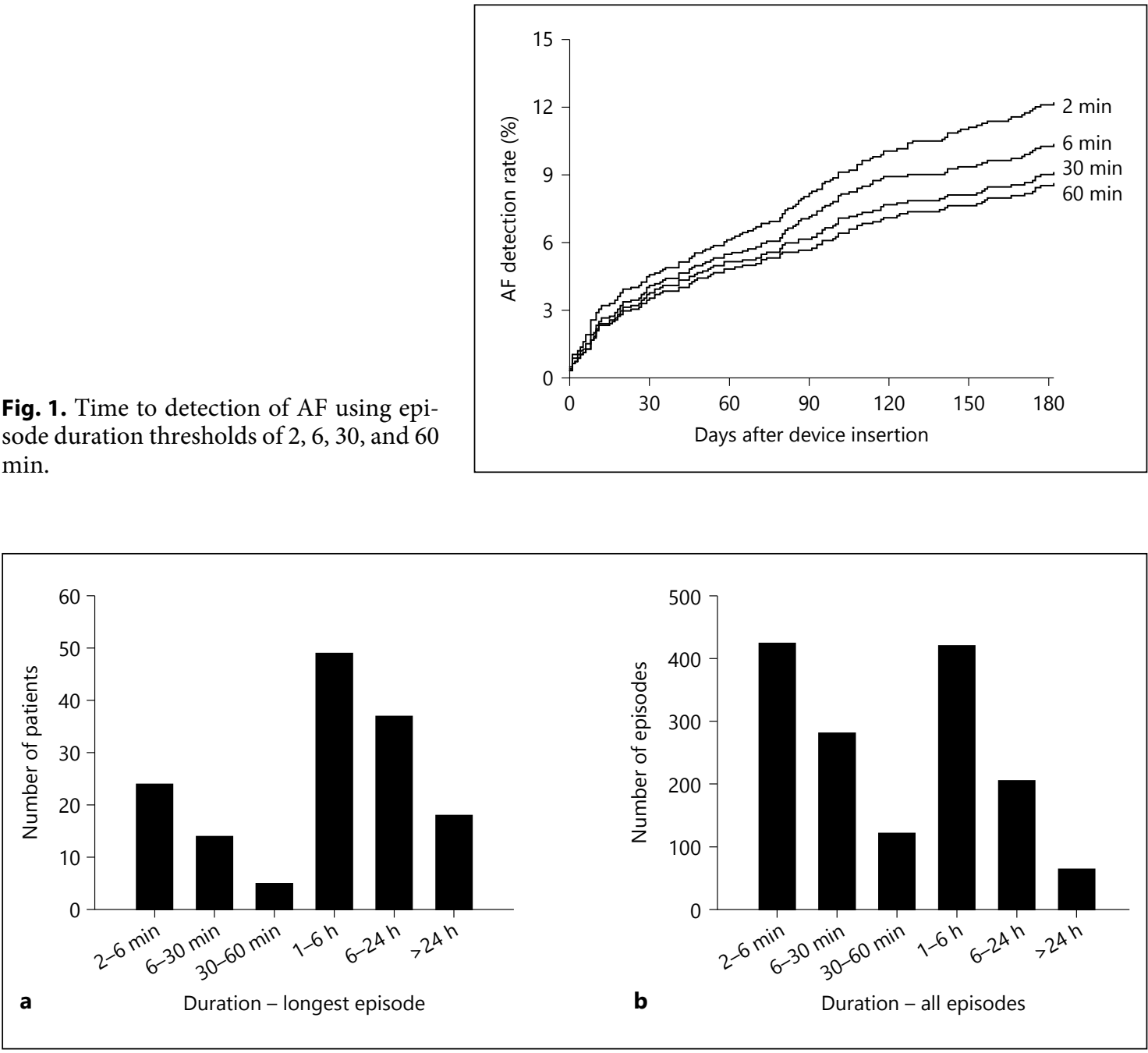

Fig. 2. Distribution of maximum episode durations among 147 patients with AF detected (a) and all episode durations among 1,521 episodes detected (b).

Discrete variables are reported as counts and percentages and continuous variables are reported as mean $\pm \mathrm{SD}$ or median (IQR), as appropriate. $p$ values $<0.05$ were considered statistically significant and all analyses were performed with SAS software version 9.2 (SAS Institute, Cary, N.C., USA).

\section{Results}

A total of 1,247 patients were included in the study and followed for a median of 182 (IQR 182-182) days. The mean age of the population was $65.3 \pm 13.0$ years and $53 \%$ were male. The ICM detected a total of $1521 \mathrm{AF}$ episodes in 147 patients. A single AF episode was detected in 42 patients (29\%), while multiple AF episodes were detected in 105 patients $(71 \%)$.

Incidence of AF in Cryptogenic Stroke

\section{AF Detection Rates}

At 1 month of follow-up (30 days), the AF detection rates were $4.6,4.1,3.8$, and $3.5 \%$ for episode duration thresholds of 2, 6, 30, and $60 \mathrm{~min}$, respectively. By 6 months of follow-up (182 days), the AF detection rates increased to $12.2,10.4,9.1$, and $8.6 \%$ for episode duration thresholds of 2, 6, 30, and $60 \mathrm{~min}$, respectively (fig. 1). The median time to detection of the first adjudicated AF episode was 58 (IQR 11-101) days.

\section{Longest Episode Duration}

The distribution of the longest-detected AF episode durations is presented in figure $2 \mathrm{a}$. In this cryptogenic stroke population, $70.7 \%$ of patients with AF detected had at least 1 episode greater than $1 \mathrm{~h}$ in duration, $37.4 \%$ had 
at least 1 episode greater than $6 \mathrm{~h}$ in duration, and $12.2 \%$ had at least 1 episode greater than $24 \mathrm{~h}$ in duration. The median duration of the longest detected AF episode was 3.4 (IQR 0.4-11.8) h. Among all 1,521 detected episodes of $\mathrm{AF}$, the mean and median duration were $4.65 \pm 13.28$ and 0.70 (IQR 0.10-3.80) h, respectively. The distribution of all episode durations is presented in figure $2 \mathrm{~b}$.

\section{Impact of Age on AF Status and Duration}

Patients who had AF episodes detected by the ICM were significantly older than patients without AF detected (71.3 \pm 10.9 vs. $64.5 \pm 13.1$ years, respectively; $\mathrm{p}<0.001$ ). The AF detection rate among patients $\leq 60$ years of age was $6 \%$ compared to $15 \%$ for patients $>60$ years $(p<0.001)$. However, among patients with AF detected, age did not differ significantly between those having a longest episode duration of $<1,1-4,4-12$, or $>12 \mathrm{~h}(\mathrm{p}=0.20)$.

\section{Comparison to CRYSTAL AF}

At 6 months of follow-up, the AF detection rate in the CRYSTAL AF clinical study was $8.9 \%$ [18] compared to an AF detection rate of $12.2 \%$ in this analysis of realworld clinical practice. This represents a $37 \%$ relative increase in the rate of AF detection over CRYSTAL AF at the 6 month time-point.

\section{Discussion}

The main finding of this study is that the AF detection rate in patients with an ICM placed following a cryptogenic stroke in real-world practice was $4.6 \%$ at 1 month and this substantially increased to $12.2 \%$ by 6 months, exceeding the rate of detection reported in CRYSTAL AF by $37 \%$ on a relative basis. Very few patients had only brief episodes of AF and patients with AF detected were older than those without AF detected.

\section{AF Detection Rates}

We observed AF detection rates of 4.6 and $12.2 \%$ at 1 and 6 months, respectively. Consequently, $62 \%$ of those with AF detected (7.6\% of our entire population) could potentially have eluded an AF diagnosis under the current guideline recommendation for up to 30 days of cardiac rhythm monitoring in stroke patients with no apparent cause [12]. In fact, the median time to detection of the first AF episode was nearly twice (58 days) the guideline recommendation and might be impractical to accomplish with prolonged use of external monitors due to patient compliance concerns $[22,23]$. This is consistent with oth- er studies of stroke patients carried out utilizing longterm continuous monitoring; these studies have shown that the majority of AF episodes are detected well beyond 30 days $[18,24]$. The proportion of patients experiencing AF who are missed by limiting the duration of monitoring to 30 days in our cohort will only continue to grow as additional follow-up information beyond 6 months is accumulated. In light of this emerging data from long-term continuous monitors, it may be reasonable to re-evaluate the current guidelines to ensure that a greater proportion of patients who may benefit from oral anticoagulation for secondary stroke prevention are identified.

The EMBRACE study reported an AF detection rate among patients randomized to the external event recorder arm of $16.1 \%$ at 90 days [25], which is higher than our observed AF detection rate of $8.2 \%$ over the same timeframe. This dissimilarity may be partially attributable to differences in the minimum AF episode duration requirement between the 2 studies (30 s in EMBRACE [25] vs. 2 min in our study). When only episodes longer than 2.5 min were considered in EMBRACE, the AF detection rate at 90 days dropped to a more similar rate of $9.9 \%$ [25].

\section{Impact of Episode Duration}

While an expert consensus statement on AF ablation has suggested that $30 \mathrm{~s}$ of AF constitutes an AF episode [26], the clinical relevance of such extremely brief episodes is not fully understood. Part of the rationale for the $30 \mathrm{~s}$ criteria is the assumption that if such brief episodes are detected clinically with intermittent monitoring, then patients are likely to have more significant periods of AF when their rhythm is not being monitored. With continuous monitoring via implantable devices, these 'significant' periods of AF will not be missed and thus the detection of extremely brief harbinger episodes may be less critical. Still, there is ongoing debate about the amount of device-detected AF required to elevate primary stroke risk with thresholds ranging from $5 \mathrm{~min}$ in an ancillary study of the MOST trial [27], 6 min in the ASSERT study [28], $1 \mathrm{~h}$ in the SOS-AF investigation [29], $5.5 \mathrm{~h}$ in the TRENDS trial [30], to $24 \mathrm{~h}$ in the AT500 Registry [31]. However, the duration of AF episodes may be less critical in secondary stroke prevention since all patients have a $\mathrm{CHADS}_{2}$ or $\mathrm{CHA}_{2} \mathrm{DS}_{2}$-VASc score of at least 2 conferred by their prior stroke and therefore are already at an elevated risk of a recurrent stroke.

In our study, we employed a minimum episode duration threshold of 2 min due to the requirement for the $\mathrm{AF}$ detection algorithm to accrue ventricular irregularity evidence over this timeframe. Nevertheless, a majority of 
patients with at least 2 min of AF detected also had episodes of at least 6,30 , and $60 \mathrm{~min}$ in duration (fig. 1). Half the number of patients with detected AF episodes experienced an AF episode of at least $3.4 \mathrm{~h}$ in duration and almost one-quarter experienced an episode of at least $12 \mathrm{~h}$ in duration. In the CRYSTAL AF study of patients with a recent cryptogenic stroke (which also employed devices having a 2 min detection threshold), it was observed that 97\% of patients with AF detected were prescribed oral anticoagulation by their treating physician at 1 year [18]. While we were unable to collect anticoagulation usage in our de-identified database, it is likely that oral anticoagulation was prescribed at an equally high rate, given the similarities in patient populations and treating physicians. In the EMBRACE trial, the use of oral anticoagulation increased 3-fold in the group that underwent monitoring with a 30-day event recorder and resulted in significantly higher overall anticoagulation utilization compared to the control group ( 18.6 vs. $11.1 \%, \mathrm{p}=0.01$ ) [25].

\section{Impact of Age on AF Incidence}

We found that cryptogenic stroke patients in whom AF was detected were significantly older than those without AF detected. This finding is well appreciated in both the general population [32] and patients with prior stroke [33], but has typically been limited to AF, that has been discovered clinically through the evaluation of patient symptoms or intermittent external monitoring. Our study extends these findings to device-detected AF in the cryptogenic stroke population. This is particularly important since the benefits of oral anticoagulation have been shown to increase among older patients [34] and therefore age may be a factor to help identify patients who would receive the greatest benefit from more intensive arrhythmia monitoring. This also highlights the importance of considering factors such as age when comparing the rates of AF detection between different studies [35]. For example, some of the difference in $\mathrm{AF}$ detection rates between the recent CRYSTAL AF [18] and EMBRACE [25] studies may be attributed to the 12-year age difference between the 2 populations.

\section{Comparison to CRYSTAL AF}

We observed a $37 \%$ relative increase in the detection rate of $\mathrm{AF}$ at 6 months compared to the CRYSTAL AF study (12.2 vs. $8.4 \%)$. As discussed earlier, patient age is an important factor that influences the likelihood of detecting AF. The average age in CRYSTAL AF was $61 \pm 11$ years [18], while the average age in our cohort was $65 \pm 13$

Incidence of AF in Cryptogenic Stroke years. Lack of comprehensive demographic or neurologic imaging [36] data in our study precludes further exploration of additional parameters, which may have also contributed to the differences in AF detection rates between the 2 studies.

All patients in CRYSTAL AF underwent a rigorous stroke work-up, which included at least $24 \mathrm{~h}$ of arrhythmia monitoring, a transesophageal echocardiogram, screening for hypercoagulable states (in patients $<55$ years), and imaging of the head and neck. In contrast, the work-up prior to deeming a stroke cryptogenic in our study was left to the discretion of the treating physician and may not have included all of these tests. Therefore, some patients included in our study who were found to have AF might have been excluded under the CRYSTAL AF criteria.

\section{Strengths and Limitations}

The strengths of this study include its large number of patients, comprehensive follow-up with continuous rhythm monitoring, and representation of real-world clinical practice. To our knowledge, this is the largest cohort of cryptogenic stroke patients monitored continuously for AF with ICMs. Nearly all patients were followed for 6 months and this allowed for a direct comparison to the primary endpoint of the CRYSTAL AF study [18]. However, although CRYSTAL AF employed the strict inclusion/exclusion criteria of a rigorously controlled clinical trial, this analysis better reflects how physicians are using ICMs to monitor cryptogenic stroke patients in real-world clinical practice.

Limitations of the study include few demographic data, including no medication or stroke outcomes data, due to the de-identified nature of the database. Furthermore, the precise date of the index cryptogenic stroke is unknown, although it is our experience that device insertion typically occurs within the hospitalization period for the stroke event or shortly thereafter. It is possible that we underestimated the incidence of AF since very brief episodes $(<2 \mathrm{~min}$ ) may have been missed due to the AF detection algorithm requirements. However, the clinical relevance of such extremely brief episodes is not known and many patients with extremely brief episodes would still have been detected, albeit with a slight delay, upon experiencing a subsequent longer episode. The lack of control over the stroke work-up may have contributed to the higher rate of detection compared to CRYSTAL AF due to potentially less inpatient telemetry monitoring for AF or less frequent use of transesophageal echocardiogram to rule out cardiac causes. 


\section{Conclusions}

This real-world study of AF incidence among patients being monitored with an ICM after a cryptogenic stroke validates the findings of CRYSTAL AF and indicates that AF in the cryptogenic stroke population may be even more common in clinical practice than in rigorously controlled clinical studies. The majority of patients with AF episodes detected were identified more than 30 days after the initiation of monitoring, suggesting that continuous monitoring for periods longer than the current guideline recommendation of 30 days may be warranted in the evaluation of patients with cryptogenic stroke.

\section{Sources of Funding}

This study was funded by Medtronic.

\section{Disclosure Statement}

P.D.Z.: employee and shareholder (Medtronic); J.D.R.: consultant (Medtronic, Biotronik); speaker's bureau (Medtronic, Biotronik); S.W.F.: consultant (St. Jude Medical); speaker's bureau (Medtronic); A.J.N.: shareholder (Boston Scientific, Medtronic); S.S.: employee and shareholder (Medtronic); J.L.K.: employee and shareholder (Medtronic); E.N.W.: employee and shareholder (Medtronic); M.R.: speaker's bureau (Biotronik, Medtronic, Boston Scientific, Boehringer Ingelheim, Janssen, Pfizer, Bristol Myers Squibb); consultant (Biotronik, Boston Scientific).

\section{References}

1 Mozaffarian D, Benjamin EJ, Go AS, Arnett DK, Blaha MJ, Cushman M, et al: Heart disease and stroke statistics - 2015 update: a report from the American Heart Association. Circulation 2015;131:e29-e322.

2 Kolominsky-Rabas PL, Weber M, Gefeller O, Neundoerfer B, Heuschmann PU: Epidemiology of ischemic stroke subtypes according to TOAST criteria: incidence, recurrence, and long-term survival in ischemic stroke subtypes: a population-based study. Stroke 2001; 32:2735-2740.

3 Strickberger SA, Ip J, Saksena S, Curry K, Bahnson TD, Ziegler PD: Relationship between atrial tachyarrhythmias and symptoms. Heart Rhythm 2005;2:125-131.

4 Quirino G, Giammaria M, Corbucci G, Pistelli P, Turri E, Mazza A, et al: Diagnosis of paroxysmal atrial fibrillation in patients with implanted pacemakers: relationship to symptoms and other variables. Pacing Clin Electrophysiol 2009;32:91-98.

5 Page RL, Wilkinson WE, Clair WK, McCarthy EA, Pritchett EL: Asymptomatic arrhythmias in patients with symptomatic paroxysmal atrial fibrillation and paroxysmal supraventricular tachycardia. Circulation 1994;89: 224-227.

6 Ziegler PD, Koehler JL, Mehra R: Comparison of continuous versus intermittent monitoring of atrial arrhythmias. Heart Rhythm 2006;3:1445-1452.

7 Charitos EI, Stierle U, Ziegler PD, Baldewig M, Robinson DR, Sievers HH, et al: A comprehensive evaluation of rhythm monitoring strategies for the detection of atrial fibrillation recurrence: insights from 647 continuously monitored patients and implications for monitoring after therapeutic interventions. Circulation 2012;126:806-814.

8 Granger CB, Alexander JH, McMurray JJ, Lopes RD, Hylek EM, Hanna M, et al: Apixa- ban versus warfarin in patients with atrial fibrillation. N Engl J Med 2011;365:981-992.

9 Patel MR, Mahaffey KW, Garg J, Pan G, Singer DE, Hacke W, et al: Rivaroxaban versus warfarin in nonvalvular atrial fibrillation. $\mathrm{N}$ Engl J Med 2011;365:883-891.

10 Connolly SJ, Ezekowitz MD, Yusuf S, Eikelboom J, Oldgren J, Parekh A, et al: Dabigatran versus warfarin in patients with atrial fibrillation. N Engl J Med 2009;361:1139-1151.

11 Giugliano RP, RuffCT, Braunwald E, Murphy SA, Wiviott SD, Halperin JL, et al: Edoxaban versus warfarin in patients with atrial fibrillation. N Engl J Med 2013;369:2093-2104.

12 Kernan WN, Ovbiagele B, Black HR, Bravata DM, Chimowitz MI, Ezekowitz MD, et al: Guidelines for the prevention of stroke in patients with stroke and transient ischemic attack: a guideline for healthcare professionals from the American Heart Association/ American Stroke Association. Stroke 2014;45: 2160-2236.

13 Ritter MA, Kochhäuser S, Duning T, Reinke F, Pott C, Dechering DG, et al: Occult atrial fibrillation in cryptogenic stroke: detection by 7-day electrocardiogram versus implantable cardiac monitors. Stroke 2013;44:1449-1452.

14 Cotter PE, Martin PJ, Ring L, Warburton EA, Belham M, Pugh PJ: Incidence of atrial fibrillation detected by implantable loop recorders in unexplained stroke. Neurology 2013;80: 1546-1550.

15 Etgen T, Hochreiter M, Mundel M, Freudenberger T: Insertable cardiac event recorder in detection of atrial fibrillation after cryptogenic stroke: an audit report. Stroke 2013;44: 2007-2009.

16 Rojo-Martinez E, Sandín-Fuentes M, CallejaSanz AI, Cortijo-García E, García-Bermejo P, Ruiz-Piñero M, et al: [High performance of an implantable Holter monitor in the detection of concealed paroxysmal atrial fibrillation in patients with cryptogenic stroke and a suspected embolic mechanism]. Rev Neurol 2013;57:251-257.

17 Christensen LM, Krieger DW, Højberg S, Pedersen OD, Karlsen FM, Jacobsen MD, et al: Paroxysmal atrial fibrillation occurs often in cryptogenic ischaemic stroke. Final results from the SURPRISE study. Eur J Neurol 2014; 21:884-889.

18 Sanna T, Diener HC, Passman RS, Di Lazzaro V, Bernstein RA, Morillo CA, et al: Cryptogenic stroke and underlying atrial fibrillation. N Engl J Med 2014;370:2478-2486.

19 Sarkar S, Ritscher D, Mehra R: A detector for a chronic implantable atrial tachyarrhythmia monitor. IEEE Trans Biomed Eng 2008;55: 1219-1224.

20 Hindricks G, Pokushalov E, Urban L, Taborsky M, Kuck KH, Lebedev D, et al: Performance of a new leadless implantable cardiac monitor in detecting and quantifying atrial fibrillation: results of the XPECT trial. Circ Arrhythm Electrophysiol 2010;3:141-147.

21 Pürerfellner $H$, Pokushalov E, Sarkar S, Koehler J, Zhou R, Urban L, et al: P-wave evidence as a method for improving algorithm to detect atrial fibrillation in insertable cardiac monitors. Heart Rhythm 2014;11:1575-1583.

22 Vasamreddy CR, Dalal D, Dong J, Cheng A, Spragg D, Lamiy SZ, et al: Symptomatic and asymptomatic atrial fibrillation in patients undergoing radiofrequency catheter ablation. J Cardiovasc Electrophysiol 2006;17:134-139.

23 Kamel H, Navi BB, Elijovich L, Josephson SA, Yee AH, Fung G, et al: Pilot randomized trial of outpatient cardiac monitoring after cryptogenic stroke. Stroke 2013;44:528-530.

24 Ziegler PD, Glotzer TV, Daoud EG, Wyse DG, Singer DE, Ezekowitz MD, et al: Incidence of newly detected atrial arrhythmias via implantable devices in patients with a history of thromboembolic events. Stroke 2010;41:256-260. 
25 Gladstone DJ, Spring M, Dorian P, Panzov V, Thorpe KE, Hall J, et al: Atrial fibrillation in patients with cryptogenic stroke. N Engl J Med 2014;370:2467-2477.

26 Calkins H, Kuck KH, Cappato R, Brugada J, Camm AJ, Chen SA, et al: 2012 HRS/EHRA/ ECAS expert consensus statement on catheter and surgical ablation of atrial fibrillation: recommendations for patient selection, procedural techniques, patient management and follow-up, definitions, endpoints, and research trial design. Heart Rhythm 2012;9:632-696.

27 Glotzer TV, Hellkamp AS, Zimmerman J, Sweeney MO, Yee R, Marinchak R, et al: Atrial high rate episodes detected by pacemaker diagnostics predict death and stroke: report of the Atrial Diagnostics Ancillary Study of the mode selection trial (MOST). Circulation 2003;107:1614-1619.

28 Healey JS, Connolly SJ, Gold MR, Israel CW, Van Gelder IC, Capucci A, et al: Subclinical atrial fibrillation and the risk of stroke. $\mathrm{N}$ Engl J Med 2012;366:120-129.
29 Boriani G, Glotzer TV, Santini M, West TM, De Melis M, Sepsi M, et al: Device-detected atrial fibrillation and risk for stroke: an analysis of $>10,000$ patients from the SOS AF project (stroke prevention strategies based on atrial fibrillation information from implanted devices). Eur Heart J 2014;35:508-516.

30 Glotzer TV, Daoud EG, Wyse DG, Singer DE, Ezekowitz MD, Hilker C, et al: The relationship between daily atrial tachyarrhythmia burden from implantable device diagnostics and stroke risk: the TRENDS study. Circ Arrhythm Electrophysiol 2009;2:474-480.

31 Capucci A, Santini M, Padeletti L, Gulizia M, Botto G, Boriani G, et al: Monitored atrial fibrillation duration predicts arterial embolic events in patients suffering from bradycardia and atrial fibrillation implanted with antitachycardia pacemakers. J Am Coll Cardiol 2005;46:1913-1920.

32 Go AS, Hylek EM, Phillips KA, Chang Y, Henault LE, Selby JV, et al: Prevalence of diagnosed atrial fibrillation in adults: national implications for rhythm management and stroke prevention: the anticoagulation and risk factors in atrial fibrillation (ATRIA) study. JAMA 2001;285:2370-2375.

33 Wachter R, Weber-Krüger M, Seegers J, Edelmann F, Wohlfahrt J, Wasser K, et al: Agedependent yield of screening for undetected atrial fibrillation in stroke patients: the FindAF study. J Neurol 2013;260:2042-2045.

34 van Walraven C, Hart RG, Connolly S, Austin PC, Mant J, Hobbs FD, et al: Effect of age on stroke prevention therapy in patients with atrial fibrillation: the atrial fibrillation investigators. Stroke 2009;40:1410-1416.

35 Glotzer TV, Ziegler PD: Cryptogenic stroke: is silent atrial fibrillation the culprit? Heart Rhythm 2015;12:234-241.

36 Bernstein RA, Di Lazzaro V, Rymer MM, Passman RS, Brachmann J, Morillo CA, et al: Infarct topography and detection of atrial fibrillation in cryptogenic stroke: results from CRYSTAL AF. Cerebrovasc Dis 2015;40:9196. 